\title{
Captivity effects on wide-ranging carnivores
}

Animals that roam over a large territory in the wild do not take kindly to being confined.

S ome species - ring-tailed lemurs and snow leopards, for example - apparently thrive in captivity, whereas others, such as Asian elephants and polar bears, are prone to problems that include poor health, repetitive stereotypic behaviour and breeding difficulties. Here we investigate this previously unexplained variation in captive animals' welfare by focusing on caged carnivores, and show that it stems from constraints imposed on the natural behaviour of susceptible animals, with wide-ranging lifestyles in the wild predicting stereotypy and the extent of infant mortality in captivity. Our findings indicate that the keeping of naturally wide-ranging carnivores should be either fundamentally improved or phased out.

Preventing natural behaviour patterns in animals can give rise to stress and frustration $^{1,2}$, and impair the development of brain regions that are involved in behavioural sequencing, thereby reducing the animal's ability to behaveflexiblyandappropriately $y^{3,4}$. To investigate whether the observed variation in the welfare of different species could arise from a differential impact of captivity on their natural behaviour, wecalculated the mean frequency of stereotypic pacing ${ }^{5}$ by 35 species of caged carnivore. We focused on pacing because it is the most prevalent stereotypy among carnivores $(97 \%$ of reported stereotypies ${ }^{5}$ ) and also to avoid comparability problems raised by pooling different forms of stereotypy (such as swaying and head-nodding). We also quantified infant mortality in captivity, which is often dueto poor maternal care ${ }^{6}$.

Asan animal'snatural ranging and foraging activities are particularly constrained by captivity ${ }^{7}$, we obtained all available field dataon median home rangesize, daily travel distance, time spent in general activity, time spent foraging, and reliance on hunting. We also quantified minimum home-range sizes and daily travel distances, as these can be orders of magnitude smaller when food is abundant ${ }^{8}$. Relationships between wild and captive variables were tested by using one-tailed regressions.

Body-weight effects were investigated in analyses involving range size ${ }^{9}$; phylogenetic effectswerecontrolled wherenecessary (and in all analyses involving body mass) by comparative analysis of independent contrasts ${ }^{10,11}$. O ur inferences about welfare took into account natural infant-mortality rates, and theamount of normal activity and total stereotypy in captivity; we also considered feeding regimes, and the sizeand complexity of enclosures, to check that relationships between wild and captive variables were

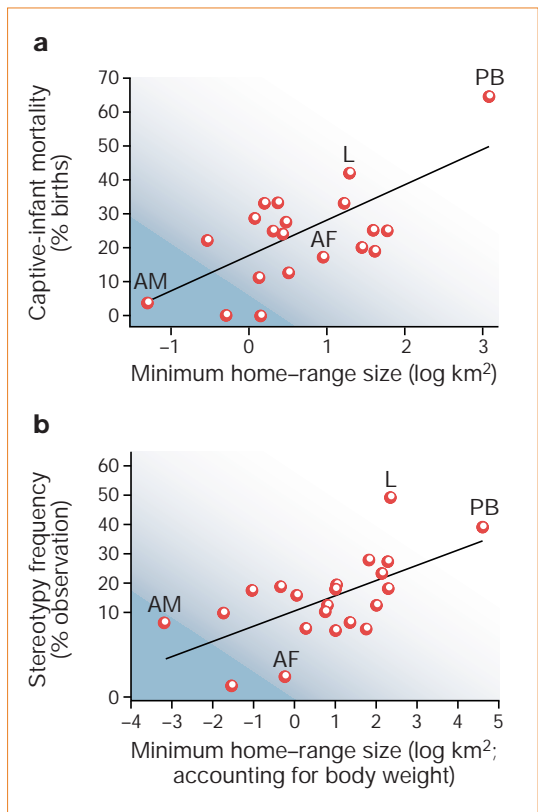

Figure 1 Natural ranging behaviour and welfare of species from the order Carnivora in captivity. a, Carnivores' minimum home-range sizes in the wild predict captive infant mortality $\left(F_{1,19}=12.60, P=0.001\right)$. b, Together with body weight (see text), minimum home-range size also predicts stereotypic pacing in captivity $\left(F_{2,19}=4.79, P=0.011\right.$; controlling for phylogeny: $\left.F_{2,17}=3.11, P=0.036\right)$. On these cross-species plots, a few species from a range of families and with varying relation to the regression line are highlighted: AF, Arctic fox (Alopex lagopus); PB, polar bear (Ursus maritimus); AM, American mink (Mustela vison); $\mathrm{L}$, lion (Panthera leo). Values on the $x$-axes differ because fitted values are used in $\mathbf{b}$ that incorporate body weight; the $y$-axis in $\mathbf{b}$ shows data back-transformed from an arc-sine transformation.

not by-products of variation in husbandry. Degrees of freedom varied in subsequent analysesowing to missing data.

Natural home-range size (HR) predicted captive-infant mortality (median $\mathrm{HR}$ : $F_{1,21}=6.04, P=0.012$; minimum $H R$, seeFig. 1a). Controlling for body weight did not alter this relationship (median HR: $F_{1,20}=4.35$, $P=0.025$; controlling for phylogeny: $F_{1,16}=20.46, \quad P=0.0001$; minimum $H R$ : $F_{1,18}=9.29, P=0.004$; controlling for phylogeny: $\left.F_{1,18}=16.94, P=0.001\right)$. M inimum, but not median, daily distances travelled (DDT) gave similar results $\left(F_{1,18}=3.99\right.$, $P=0.03)$. These effects seem to bespecific to captive animals: wild and captive infantmortality rates did not covary $\left(F_{1,6}=0.08\right.$, not significant) and infant mortality in the wild was unrelated to range size (for example, minimum HR: $F_{1,7}=0.43$, n.s.) .

Home-range size also predicted pacing (median $H R: F_{122}=5.78, P=0.013$; minimum HR: $\left.F_{1,20}=5.66, P=0.014\right)$. A positive trend was evident with body weight $\left(F_{1,33}=3.23, P=0.081\right.$; controlling for phylogeny: $\left.F_{1,31}=4.09, P=0.052\right)$. With both terms in a multiple regression, each lost its individual effect on pacing, but the overall adjusted $r^{2}$ value increased to $26.5 \%$, from $6.2 \%$ (for body weight alone) and $18.2 \%$ (for minimum HR alone; Fig. 1b). Likewise, median, but not minimum, daily travel distanceswere positively correlated with pacing $\left(F_{1,18}=9.80, P=0.003\right)$.

These results all held when total stereotypical behaviours (including non-pacing) were analysed ${ }^{5}$. Naturally wide ranging animals did not, however, show more normal activity in captivity (for example, minimum HR: $F_{1,15}=0.17$, n.s.; DDT: $F_{1,15}=0.01$, n.s.) nor did they move around more overall within their enclosures (for example, minimum HR: $F_{1,18}=0.61$, n.S.; DDT: $F_{1,18}=0.10$, n.s.). Home-range size therefore still predicted pacing, even when controlling for the amount of total activity in captivity (for example, minimum HR: $F_{1,14}=2.65$, $P=0.019)$. The degree of natural foraging and general activity, in contrast, did not predict captive stereotypy or infant mortality ${ }^{5}$ (for example, the level of natural activity versus pacing: $F_{1,18}=3.53$, n.s.). Variations in husbandry did not account for any of thesefindings ${ }^{5}$.

Our results show, to our knowledge for thefirst time, that a particular lifestylein the wild confers vulnerability to welfare problems in captivity. Our study also reveals species that areinherently likely to farebadly in zoos and similar establishments. Among the carnivores, naturally wide-ranging species show the most evidence of stress and/or psychological dysfunction in captivity $^{3,4,12}$, a finding that is a cause for concern, given the difficulties of conserving such species in situ ${ }^{13}$. Husbandry of these species in captivity is therefore in need of improvement, such as provision of extra space (a polar bear'stypical enclosure size, for example, is about one-millionth of its minimum home-range size). Alternatively, zoos could stop housing wide-ranging carnivores and concentrate instead on species that respond better to being kept in captivity.

\section{Ros Clubb, Georgia Mason}

Animal Behaviour Research Group, Department of Zoology, University of Oxford, South Parks Road, Oxford OX1 3PS, UK

e-mail: georgia.mason@zoology.ox.ac.uk

1. Mason, G., Cooper, J. \& Clarebrough, C. Nature 410, 35-36 (2001).

2. Dawkins, M. S. Appl. Anim. Behav. Sci. 20, 209-225 (1988).

3. Robbins, T. W., Jones, G. H. \& Wilkinson, L. S.

J. Psychopharmacol. 10, 39-47 (1996).

4. Lewis, M. H., Gluck, J. P., Bodfish, J. W., Beauchamp, A. J. \& 
Mailman, R. B. in Stereotyped M ovements (eds Sprague, R. L. \& Newell, K. M.) 37-67 (Am. Psychol. Assoc., Washington DC, 1996)

5. Clubb, R. E. The Roles of Foraging N iche, Rearing Conditions and Current Husbandry on the D evelopment of Stereotypies in Carnivores (Thesis, Univ. Oxford, 2001).

6. Meier, J. in Zoo and Wild Animal M edicine (ed. Fowler, M . E.) 842-851 (Saunders, Philadelphia, 1986).

7. Shepherdson, D. J., M ellen, J. D. \& Hutchins, M . (eds) Second Nature: Environmental Enrichment for Captive Animals (Smithsonian Inst. Press, Washington DC, 1998)

8. Gittleman, J. L. \& Harvey, P. H. Behav. Ecol. Sociobiol. 10, 57-63 (1982)

9. Gittleman, J. L. J. M amm. 67, 23-36 (1986).

10. Purvis, A. \& Rambaut, A. Comp. Appl. Biosci. 11, 247-251 (1995).

11. Bininda-Emonds, O. R. P., Gittleman, J. L. \& Purvis, A. Biol. Sci 74, 143-175 (1999).

12. Bahr, N. I., Pryce, C. R., Döbeli, M . \& Martin, R. D. Physiol. Behav. 64, 429-437 (1998)

13. Woodroffe, R. \& Ginsberg, J. R. Science 280, 2126-2128 (1998) Competing financial interests: declared (see online version).

\section{Explosives}

\section{A microsensor for trinitrotoluene vapour}

S ensing devices designed to detect explosive vapours are bulky, expensive and in need of technological improvement - dogs remain the most effective detectors ${ }^{1}$ in the fight against terrorism and in the removal of land-mines ${ }^{2,3}$. Here we demonstrate the deflagration of trinitrotoluene (TNT) in a small localized explosion on an uncoated piezoresistive microcantilever. This explosive-vapour sensor, which has a detection capability that is comparable to that of a dog, should enable extremely sensitive, miniature detection devices to be used on a large scale.

Microcantilevers with specific coatings can be used for chemical detection ${ }^{4,5}$, but TNT molecules, which are intrinsically 'sticky', readily adhere to uncoated cantilever surfaces ${ }^{6}$. When the source of TNT is removed, the TNT molecules slowly desorb. We found that applying a voltage pulse to an integrated piezoresistor during this desorption process causes the cantilever temperature to rise beyond the deflagration point of TNT, resulting in a miniature deflagration. Our cantilevers did not degrade after hundreds of pulses (10-25 volts) and deflagrations.

We monitored deflagration by capturing magnified high-speed video images, by measuring the deflection due to released heat using an optical beam-bounce technique that is used in atomic-forcemicroscopy, and by measuring resonance-frequency shifts and heat added with a piezoelectric/piezoresistive cantilever ${ }^{7}$ operated in self-sensing modeby means of an a.c. bridge circuit ${ }^{8}$. The circuit's output increases with mass loading of adsorbed TNT and increasing temperature; it decreases with mass desorption and decreasing temperature.

A five-eventTNT-detection test, usingthe self-sensing platform described, is illustrated in Fig. 1. During event 1, before loading with TNT, a reference voltage pulse ( 25 volts) is applied to the piezoresistive heater, causing a temporary upward spike in circuit output that is due to heating. TNT loading (event 2) causes a gradual upward shift in sensor output, which then gradually decreases when the TNT begins to desorb from the cantilever (event 3). The second pulse (5 volts) during desorption does not raise the cantilever temperature sufficiently for deflagration (event 4). The third pulse ( 25 volts) causes deflagration, as shown by a visible smoke plume, and a dramatic mass decrease, which is verified by a reduction in circuit output (event 5) that overwhelms the upward thermal signal evident in event 1 . Post-deflagration reference pulses of 25 volts resulted in spikessimilar to theoneseen in event 1.

The occurrence of deflagration was inferred from three consistent observations. First, the cantilever returns to its pretest resonance frequency after deflagration, suggesting that all of the adsorbed material has been lost. Second, a specific voltage (corresponding to a threshold, or deflagrationpoint, temperature) is necessary to cause deflagration. Third, the measurement of heat added to the cantilever during deflagration shows that the reaction is exothermic, ruling out other possible reactions such as melting, vaporization or decomposition.

Our method currently detects the deflagration of aslittleas 70 picograms $\left(1.9 \times 10^{11}\right.$ molecules) of TNT ( calculated from theshift in cantilever resonance). Thislimit of detection is the same as that of an improved version ${ }^{9}$ of theion-mobility mass-spectrometry technology now used for airport security. Calculations show that the detection limit could be improved by up to three orders of magnitudebyusing optimized cantilevers.

An explosive-detection technique based on deflagration is advantageous, because deflagration is a characteristic property of these substances. Other potentially interfering but non-explosive substances tested including water, acetone, ethyl alcohol and gasoline - all desorbed in our system before the voltage pulse was applied. In the absence of a voltage pulse, desorption of nanogram quantities of deposited TNT takes tens of minutes; comparable amounts of water or alcohol desorb in seconds.

Other explosive molecules can be detected by this method and differentiated by their compound-specific deflagration points and desorption times (results not shown). Our techniquecould also be used in conjunction with coated-cantilever chemical-sensing arrays $^{5}$, in which the arrays serveas an initial indicator, and positive readings are verified by deflagration on an uncoated cantilever.

L. A. Pinnaduwage*t, A. Gehl*t, D. L. Hedden*†, G. Muralidharan*, T. Thundat*†, R. T. Lareauł, T. Sulchek§, L. Manning\$, B. Rogers§, M. Jones§, J. D. Adams§

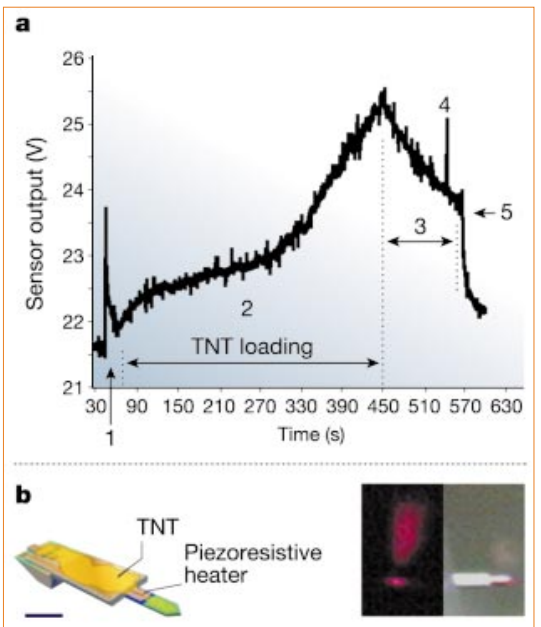

Figure 1 Deflagration and detection of trinitrotoluene (TNT). a, Sensor output plotted against time. During event 1 (numbered arrow) of the test, a 25-volt reference pulse is sent to the piezoresistor before TNT loading begins; sensor output returns quickly to almost its pre-pulse value. In event 2, TNT is gradually loaded onto the cantilever and the resonance frequency decreases, causing the sensor output to increase. After loading, the TNT begins to desorb (event 3) and a 5-volt pulse (event 4) is insufficient to heat the explosive on the cantilever to its deflagration point. However, a 25-volt pulse is sufficient to cause deflagration (event 5). b, Left, diagram of the microcantilever, showing the integrated piezoelectric sensor, piezoresistive heater, and TNT loaded on the cantilever surface. Scale bar, $100 \mu \mathrm{m}$. Right, deflagration is confirmed in magnified high-speed video images; the smoke plume is visible, particularly when illuminated by red laser light.

*Oak Ridge National Laboratory, Oak Ridge, Tennessee 37831, USA

†D epartment of Physics, University of Tennessee, Knoxville, Tennessee 37996, USA

¥Transportation Security Administration, US D epartment of H omeland Security, Atlantic City, N ew Jersey 08405, USA $\S$ D epartment of M echanical Engineering and the N evada Ventures N anoscience Program, U niversity of Nevada, Reno, Nevada 89557, USA

e-mail: jdadams@unr.edu

1. Furton, K. G. \& Myers, L. J. Talanta 54, 487-500 (2001)

2. Colton, R. J. \& Russell, J. N. Science 299, 1324-1325 (2003).

3. Czarnik, A. W. Nature 394, 417-418 (1998).

4. Thundat, T., Chen, G. Y., Warmack, R. J., Allison, D. P. \& Wachter, E. A. Anal. Chem. 67, 519-521 (1995).

5. Lang, H. et al. Appl. Phys. Lett. 72, 383 (1998).

6. Muralidharan, G. et al. Ultramicroscopy 97, 433-439 (2003)

7. Minne, S. C., Manalis, S. R. \& Quate, C. F. Appl. Phys. Lett. 67, 3918-3920 (1995)

8. Rogers, B. et al. Rev. Sci. Instruments (in the press)

9. Khayamian, T., Tabrizchi, M.\& J Jafari, M.T. Talanta 59, 327-333 (2003).

Competing financial interests: declared none.

brief communications is intended to provide a forum for brief, topical reports of general scientific interest and for technical discussion of recently published material of particular interest to non-specialist readers (communications arising). Priority will be given to contributions that have fewer than 500 words, 10 references and only one figure. Detailed guidelines are available on Nature's website (www.nature.com/nature). 